\title{
Research on Similarity of Adaptable-Function Mechanical Behavioral Process
}

\author{
Peng Yang ${ }^{1}$, Yi-min Deng ${ }^{1,2} \&$ Xi Yin ${ }^{1}$ \\ ${ }^{1}$ Faculty of Mechanical Engineering and Mechanics, Ningbo University, Ningbo ,315211, China \\ ${ }^{2}$ Zhejiang Provincial Key Lab of Part Rolling Technology, Ningbo ,315211, China
}

Keywords: adaptable-function, behavioral process, similarity, fuzzy evaluation model.

\begin{abstract}
Adaptable-function mechanical products generally demonstrate various degrees of similarity in their behavioral process for different adaptable functions. Analysis and research of this similarity constitutes an important aspect of research for the design of such products. The paper investigates the behavioral process similarity of typical adaptable-function mechanical products and summarizes the corresponding behavioral process in the behavior elements, behavior structure and behavior characteristics three level has the various degrees of similarity. For the redesign of existing products for adaptable functions and system optimization provides a theoretical basis. At last, this paper uses fuzzy evaluation method to construct a fuzzy comprehensive evaluation model to evaluate the similarity of behavioral process.
\end{abstract}

\section{Introduction}

In recent years, the adaptable-function mechanical products for its high comprehensive utilization rate and wide adaptability more and more welcome by consumers. Adaptable-function mechanical[1] has some characteristics of implementation system function change by replacing system components or adjust the system structure connection. Research on mechanical products redesign for adaptable-function has a positive role in promoting the sustainable development of manufacturing industry. The lack research of adaptable-function at home and abroad, especially lacks the research of concept design method for adaptable-function redesign.

Relevant data show that in product design many design is improved and changes on the basis of the original design. Product redesign kept similar with the original design[2]. Visible, use the original product characteristics to redesign another similar system is an effective way to improve the design quality and save the design cost. Research on product design method, the theory of FBS is a relatively intact theoretical system of design. Such as "function - behavior - state" [3] (FBS) design process model, and "function - behavior - structure" [4-5] (FBS) design process model. In the FBS design theory the behavior act as a bridges between function and structure, so as to obtaining the solution of the design problem. The introduction of the behavior make function expression easier to understand, also expanded the designer's creative space[6-7]. Due to the adaptable-function mechanical system is based on sharing infrastructure when function conversion, so each function system when realization its behavioral process there must be have some degree of similarity. Research on the similarity of adaptable-function mechanical system behavioral process not only can provide a certain theoretical basis for the adaptable-function mechanical system analysis, but also provide a certain theoretical guidance for the mechanical system redesign to adaptable-function mechanical system.

\section{Case study}

Mechanical products with adaptable-function can multi-usage, such as multifunctional combined tool, micro tillage machine and combination machine tool etc. These products has brought great convenience to production and life. In this paper, taking a multifunctional household cooking machine (Figure 1) which is closely linked with our life as an example to specific analysis the similarity of adaptable-function mechanical behavioral process. 


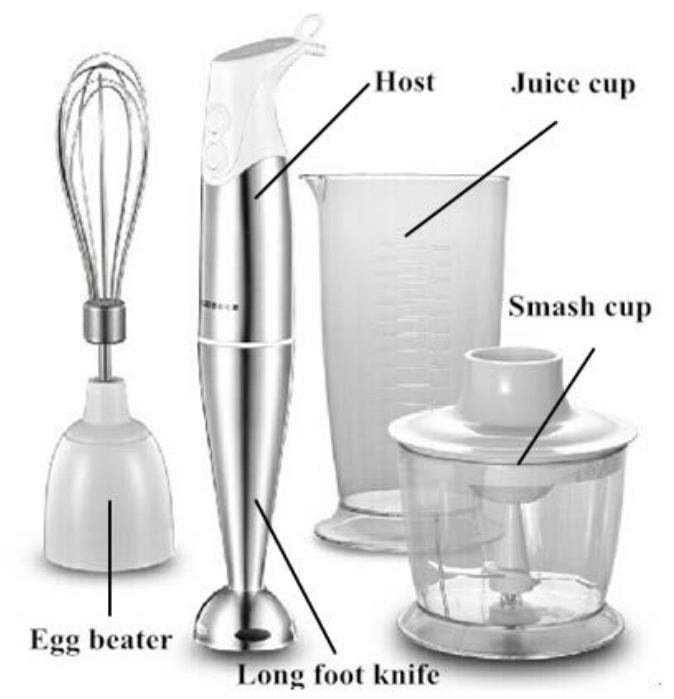

Fig.1: Multifunctional household cooking machine

The household cooking machine has the characteristics of simple structure and wide adaptability. By changing the connection of the corresponding egg beater, long foot knife and smash cup can convert multiple functions of stirred egg whites, minced meat, crushed material and make juice etc. To facilitate analysis, in this paper have analysis household cooking machine behavioral process of three kinds function[8-9]. When the host connected to the egg beater, household cooking machine has the function of stirred egg whites. The long foot knife replaced egg beater, household cooking machine has the function of make juice. The long foot knife with smash cup, cooking machine has the function of minced meat. Three kinds of behavioral process analysis are as follows:

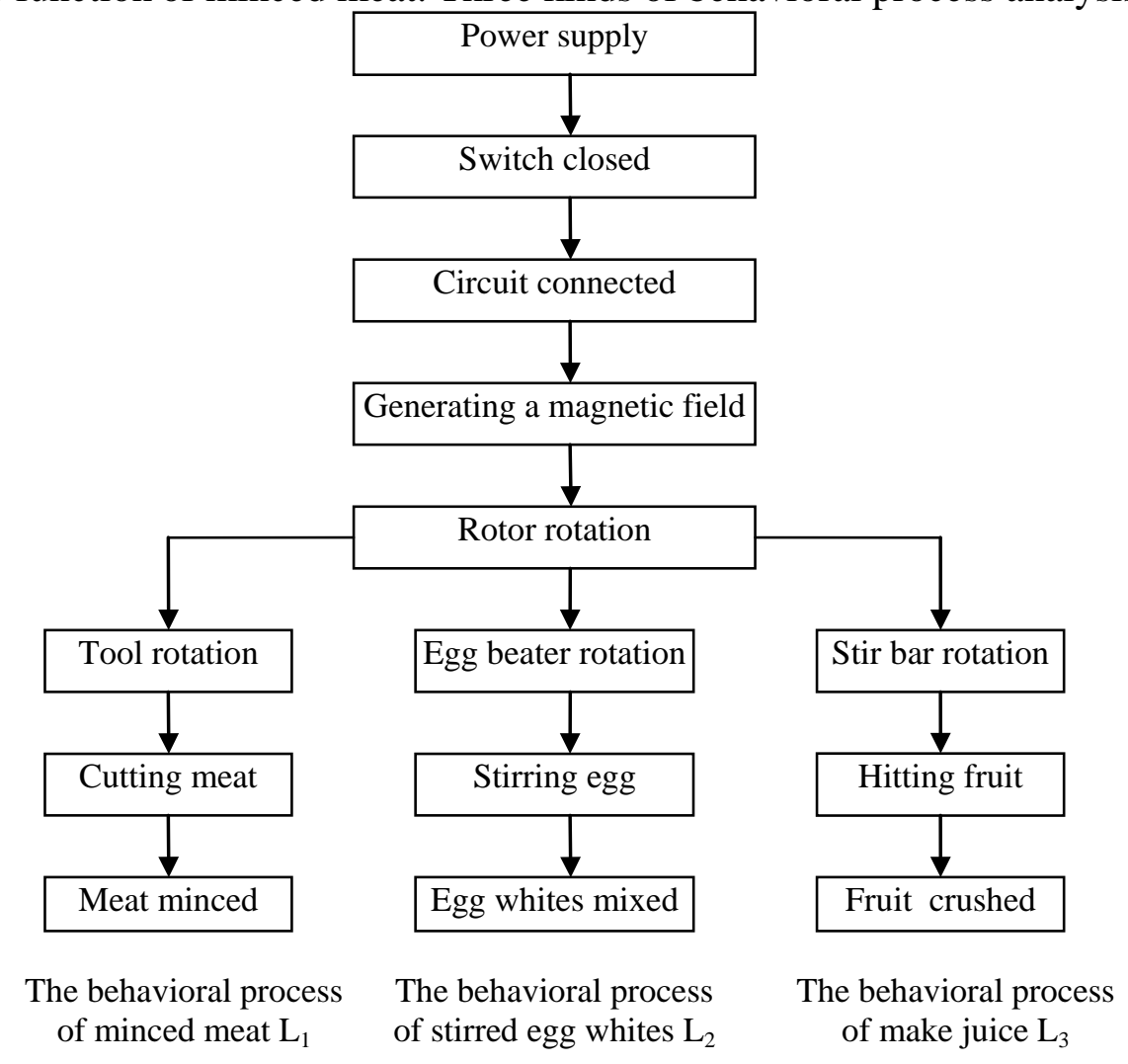

Fig.2: Three kinds of behavioral process analysis

Analysis of behavioral process from the household cooking machine three kinds of adaptablefunctions can be found in three kinds of behavioral process has the various degrees of similarity. After analysis a lot of adaptable-function mechanical products behavioral process can be found: Mechanical products with adaptable-function mapping all kinds of behavioral process has the 
various degrees of similarity in three levels of behavior elements, behavior structure and behavior characteristics.

\section{Research on similarity of behavioral process}

Analysis of behavioral process from the adaptable-function mechanical can be found behavioral process mapping in realize each function has a certain degree of similarity. It is because the behavioral process of adaptable-function mechanical each system with similarity and easy to fluent realize the adaptable-function system. We can take the behavioral process as a system which is composed of several elements with interaction relations. The system has a certain degree of similarity and regularity in three levels of behavior elements, behavior structure and behavior characteristics.

\section{The similarity of behavior elements}

Behavior elements constitute the behavioral process system. Due to the adaptable-function mechanical system is based on sharing infrastructure when function conversion, so must have some behavior elements is shared by each behavioral process. And the different parts of the behavior elements also must have similarity to some extent. The system of behavioral process can be shown in figure 2.

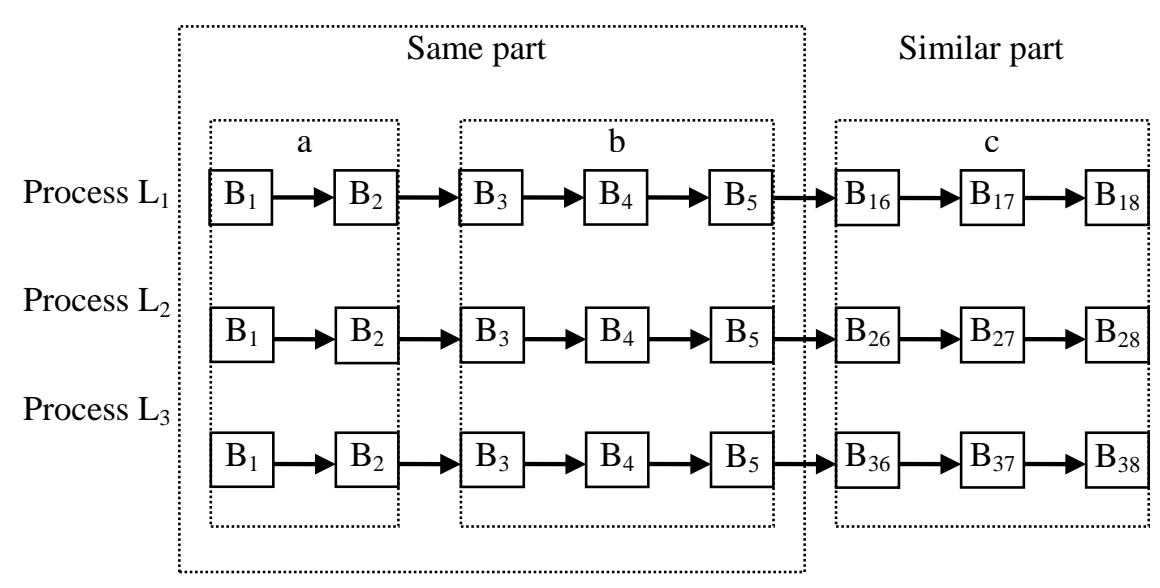

Fig.3: The behavior elements of three kinds system

\section{The similarity of behavior structure}

Behavior structure reflects the composition of behavior elements and the relationship between behavior elements in the behavioral process system. At the same time the behavior structure determines the movement and the interaction relationship between execute components. Figure 4 shows the structure model of each behavioral process. The similarity of behavior structure can provide a theoretical basis for the product structure innovation.

i)Orderly structure similarity: Elements that is composed of the behavioral process system has regularity in spatial structure arrangement and combination, also the structure between elements has good function coupling.

ii)Structure transfer similarity: That structure between the each behavior elements in the behavioral process system influence each other and transference of connection effect is similarity. 


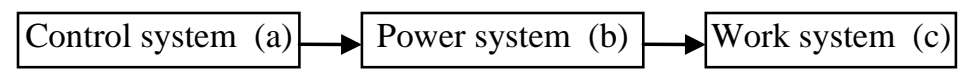

Fig.3: Behavior structure model

\section{The similarity of behavior characteristics}

Behavior characteristic is an important way to express characteristics information of function in the behavioral process. At the same time behavior characteristic is an important way to expression useful design information. Any behavioral process is composed of the relevant behavior elements, behavior characteristics also is the link between each behavior elements. Behavior characteristics expressed by each adaptable-function mechanical behavioral process system has the characteristics of causal similarity, orderly function similarity and orderly movement time similarity.

i) Causal similarity: In the behavioral process the former behavior element is a input for the after behavior element, and the two behavior elements on the energy, material and signal has the interdependent relationship.

ii)Orderly function similarity: The elements that is composed of the behavioral process system has shown the sub-functions have the certainty and regularity. The function of orderly structure system is affected by the each elements sub-functions and the sequence of function occur.

iii)Orderly movement time similarity: Elements that is composed of the behavioral process system is always according to the time sequence correlation occurred in turn and each elements having a certain movement occurrence relevance.

Because the system of adaptable-function mechanical behavioral process has a certain degree of similarity and regularity in three levels of behavior elements, behavior structure and behavior characteristics, so is easy to fluent realize the adaptable-function system. These similarities has a great theoretical guidance significance for us to optimization analysis of adaptable-function mechanical and redesign of existing products for adaptable functions.

\section{Similarity evaluation model}

For quantitative analysis the similarity of each behavioral process ,this paper uses fuzzy evaluation method[10-11] to construct a fuzzy comprehensive evaluation model.

Fuzzy comprehensive evaluation steps are as follows:

i) Determine the behavioral process element set.

$B=\left\{B_{1}, B_{2}, \cdots, B_{m}\right\}$

Where $\mathrm{Bi}$ is the $\mathrm{i}$-th element during the behavioral process.

ii) Determine the weight set

$G=\left\{g_{1}, g_{2}, \ldots, g_{n}\right\}$

Where gi is the weight of the element Bi , satisfy the conditions : $g_{i} \geq 0, \sum_{i}^{n} g_{i}=1$

iii) Determine the evaluation set.

$U=\left\{u_{1}, u_{2}, \ldots, u_{m}\right\}$ Wherein $m$ is the number of reviews, we can graded on similarity description.

iiii) Determine the fuzzy evaluation matrix of behavior element.

$$
R=\left[\begin{array}{c}
R_{1} \\
R_{2} \\
\vdots \\
R_{n}
\end{array}\right]=\left[\begin{array}{cccc}
r_{11} & r_{12} & \cdots & r_{1 m} \\
r_{21} & r_{22} & \cdots & r_{2 m} \\
\vdots & \vdots & \vdots & \vdots \\
r_{n 1} & r_{n 2} & \cdots & r_{n m}
\end{array}\right]
$$


Where rij represents the i-th element of the j-level membership evaluation.

Fuzzy comprehensive evaluation matrix considering weight coefficients:

$$
A=G \bullet R=\left(g_{1}, g_{2}, \cdots, g_{n}\right)\left[\begin{array}{cccc}
r_{11} & r_{12} & \cdots & r_{1 m} \\
r_{21} & r_{22} & \cdots & r_{2 m} \\
\vdots & & & \vdots \\
r_{n 1} & r_{n 2} & \cdots & r_{n m}
\end{array}\right]=\left(a_{1}, a_{2}, \cdots, a_{m}\right)
$$

membership of the fuzzy comprehensive evaluation set $(\mathrm{j}=1,2, \ldots, \mathrm{m})$.

$M(\wedge, \vee)$ calculation method have used to calculate. That is according to first take small $(\wedge)$ after taking large $(\vee)$,then perform matrix synthesis computing.

$$
\begin{aligned}
& \quad a_{j}=\bigvee_{i=1}^{n}\left(g_{i} \wedge r_{i j}\right) \quad(j=1,2, \cdots, m) \\
& \quad \text { Formula expand : } \\
& a_{j}=\left(g_{1} \wedge r_{1 j}\right) \vee\left(g_{2} \wedge r_{2 j}\right) \vee\left(g_{3} \wedge r_{3 j}\right) \vee \cdots\left(g_{n} \wedge r_{n j}\right) \quad(j=1,2, \cdots, m)
\end{aligned}
$$

\section{Conclusions}

Analysis of behavioral process is an important aspect of product design theory. Through the research on the similarity of typical adaptable-function mechanical behavioral process, we can from the behavior elements, behavior structure and behavior characteristics three level comprehensive analysis the behavioral process similarity between different systems. At the same time based on the similarity of the behavioral process we can provides a certain theoretical basis for the redesign of existing products for adaptable functions and system optimization. And by using the fuzzy comprehensive evaluation model can feasibility and rationality quantitative analysis the similarity of different behavioral process.

\section{Acknowledgements}

The research work was supported by National Natural Science Foundation of China under Grant No. 51375246 and Outstanding Paper nurture Foundation of Ningbo University under Grant No. py2014010.

\section{References}

[1] Yu Y W, Deng Y M, Lu W F, et al. Analysis of mechanical systems with adaptable functions for the evaluation of functional coupling and component importance [J]. The International Journal of Advanced Manufacturing Technology, 2014, doi: 10.1007/s00170-014-6358-6.

[2] Zhou Mei-li. Formation Principies of similarity between similar systems [J]. International Journal of General System, 1999, 27(6): 495-504.

[3] Umeda Y, Ishii M, Yoshioka M. Supporting conceptual design base on the function-behaviorstate modeler[J]. Artificial Intelligence in Engineering Design Analysis and Manufacturing, 1996, 10(4):275-288.

[4] Gero J S, Kannengiesser U. The situated function-behavior-structure framework [J]. Design Studies, 2004, 25 (4): 373-391.

[5] Pahl G, Beitz W, Feldhusen J, et al. Engineering Design: A Systematic Approach [M]. 3th ed. London: Springer-Verlag, 2007.

[6] Coulibaly A, Mutel B, Ait-Kadi D. Product modeling framework for behavioral performance evaluation at design stage [J]. Computers in Industry, 2007, 58(6): 567-577.

[7] Wang M Y, Shen L G, Deng Y M. The behavior of mechanical energy storage mechanisms and representation[J]. Advanced Science Letters, 2011, 4(1): 1-5.

[8] Deng Y M, Ma Y S, Wang M Y, et al. A Comprehensive Behavioural Process Model for 
Design Synthesis[J]. Future Communication, Computing, Control and Management, 2012, 141(1): 265-270.

[9] Wang M Y, Shen L G, Deng Y M. An extended causal behavioral process model for conceptual mechanical product design [J]. Computer - Aided Design and Applications, 2012, 9 (4): 419438.

[10] Ma Guo-zhong, Mi Wen-zhong. Liu Xiao-dong. Multi-level fuzzy evaluation method for civil aviation system safety[J]. Journal of Southwest Jiao tong University, 2007, 42(1): 104109.

[11] Kulak O, Kahraman C. Fuzzy multi-attribute selection among transportation companies using axiomatic design and analytic hierarchy process[J]. Information sciences, 2005, 170(2): 191-210. 